\title{
Synthesis of CNT film on the surface of micro-pyramid array and its intense pulsed emission characteristics
}

\author{
ZENG FanGuang $^{1 *}$, LI Xin $^{2}$, LIU WeiHua ${ }^{2}$, QIAO ShuZhen ${ }^{1}$, MA HuaLi ${ }^{1}$, ZHANG Rui $^{1}$, \\ XIA LianSheng ${ }^{3}$, CHEN Yi ${ }^{3}$, LIU XingGuang ${ }^{3}$ \& ZHANG Huang ${ }^{3}$ \\ ${ }^{1}$ Department of Mathematics and Physics, Zhengzhou Institute of Aeronautical Industry Management, Zhengzhou 450015, China; \\ ${ }^{2}$ School of Electronic and Information Engineering, Xi'an Jiaotong University, Xi'an 710049, China; \\ ${ }^{3}$ Institute of Fluid Physics, Chinese Academy of Engineering Physics, Mianyang 621900, China
}

Received August 15, 2011; accepted September 1, 2011; published online March 8, 2012

\begin{abstract}
We developed a new scheme to suppress the electric-field-screening effect in high growth density of a carbon nanotube (CNT) film during its intense pulsed emission. We synthesize the CNT film on a tridimensional surface (t-CNT film). The tridimensional surface includes wet etched silicon pyramids, and the Ni layer is electroless plated thereon. The intense pulsed emission characteristics of the t-CNT and planar-grown CNT (p-CNT) films were measured using a diode structure in single-pulse mode. The even turn-on field decreased from $5.5 \mathrm{~V} / \mu \mathrm{m}$ for $\mathrm{p}$-CNTs to $2.8 \mathrm{~V} / \mu \mathrm{m}$ for t-CNTs, and the peak emission current increased from $232 \mathrm{~A}$ for $\mathrm{p}$-CNTs to $324 \mathrm{~A}$ for t-CNTs at a peak field intensity $\sim 12.2 \mathrm{~V} / \mu \mathrm{m}$. The peak current of the t-CNT film increased by $\sim 39.7 \%$ over the p-CNT film. It is clear that the micro-pyramid array can effectively suppress the field screening effect to improve the electron-emission of CNT films.
\end{abstract}

carbon nanotubes, micro-pyramid array, field-screening effect, intense pulsed emission, emission current

Citation: Zeng F G, Li X, Liu W H, et al. Synthesis of CNT film on the surface of micro-pyramid array and its intense pulsed emission characteristics. Chin Sci Bull, 2012, 57: 1739-1742, doi: 10.1007/s11434-012-5028-z

Carbon nanotubes (CNTs) have attracted considerable attention for their potential field-emission applications because of their excellent properties [1-3]. These properties make CNTs a good candidate for cold cathodes in fieldemission displays, high-resolution electron-beam instruments, lamps, X-ray sources, and microwave amplifier tubes [4-9]. Improving current emission is very important for using CNTs as electron sources. However, when the density of CNTs increases, the field emission of a CNT cathode will be depressed by the field screening effect $[10,11]$, resulting in a higher turn-on voltage and lower emission current. Therefore, it is necessary to find a way to suppress the fieldscreening effect.

In this paper, we report the synthesis of a CNT film on the surface of a micro-pyramid array and its intense pulsed

*Corresponding author (email: fgzeng@ sina.com) emission characteristics (the as-grown CNT film is hereafter called the "t-CNT film"). The function of the micro-pyramid array in suppressing the field screening effect can be easily found by comparing the emission characteristics of the $\mathrm{t}-\mathrm{CNT}$ film with that of the planar-grown CNT (p-CNT) film.

\section{Experimental}

\subsection{Sample preparation}

(1) Materials and reagents. An n-type silicon ( $\mathrm{Si}$; Tianjin Institute of Semiconductor Technology, Tianjin, China) wafer (100) with a resistivity of $10^{-2}-10^{-3} \Omega \mathrm{cm}$ and 2-in diameter was used as the substrate material. We used a negative photoresistor for photolithography to transfer the designed pattern to the silicon dioxide layer. Buffered oxide 
etchants (BOE, home-made) were used for etching the silicon dioxide windows. Potassium hydroxide $(\mathrm{KOH}$; Tianjin Kaitong Chemical Reagent Co. Ltd, Tianjin, China) solution with a concentration of $40 \mathrm{wt} \%$ was used as an etchant in the anisotropic wet etching to form Si pyramids. The electroless nickel plating solution was composed of nickel sulfate (Tianjin Fengchuan Chemical Reagent Technological Co. Ltd, Tianjin, China), sodium hypophosphite $\left(\mathrm{NaH}_{2} \mathrm{PO}_{2}\right.$; Tianjin Kermel Chemical Reagent Co. Ltd, Tianjin, China), and trisodium citrate (Tianjin Kermel Chemical Reagent Co. Ltd). Palladium (II) chloride (Tianjin Kermel Chemical Reagent Co. Ltd) was used as the catalyst for the Ni plating. Iron phthalocyanine (FePc; Tokyo Chemical Reagent Co. Ltd, Tokyo, Japan) was used for growing the CNT film.

(2) Preparation procedure. Si pyramids were aligned on the Si surface by wet etching to form a tridimensional surface. The preparation of the Si pyramids was as follows. The silicon wafer was oxidized to form a silicon dioxide layer with a thickness of more than $300 \mathrm{~nm}$. We did photolithography for the resistor to transfer the designed pattern onto the silicon dioxide layer. The mask for the photolithography was made on a plastic film by a photoplotter (JOINYOU 2032A, Shenzhen, China). We etched the silicon dioxide layer to form etching windows. The etching temperature in the BOE etchants was $\sim 40^{\circ} \mathrm{C}$. We formed $\mathrm{Si}$ pyramids by anisotropic wet etching. The etching temperature of the $\mathrm{KOH}$ solution was $90-100^{\circ} \mathrm{C}$. When the Si pyramids formed, the $\mathrm{Si}$ wafer was immersed in de-ionized water and then in the BOE etchants to remove residual silicon dioxide layer.

A nickel film with $\sim 1 \mu \mathrm{m}$ thickness was electroless plated on the tridimensional surface. Before the plating of the Ni layer, the Si wafer was immersed in palladium (II) chloride aqueous solution for $30 \mathrm{~s}$. Then the catalyzed Si wafer was put into the plating solution for $5 \mathrm{~min}$ at $60^{\circ} \mathrm{C}$. The $\mathrm{pH}$ value of the solution was adjusted to be $8-10$ by adding ammonia to the solution.

The CNT film was synthesized by pyrolysis of FePc [12]. The temperature for CNT growth was $\sim 900^{\circ} \mathrm{C}$. The growth time was $\sim 10 \mathrm{~min}$.

\subsection{Study on the morphologies of samples}

A scanning electron microscope (SEM, JEOL JSM-6700F) was used to study the morphologies of the substrate and CNT film. The morphology of the tridimensional surface (Si pyramids) was observed with an optical microscope (OM).

\subsection{Measurement of intense pulsed emission charac- teristics}

We measured intense pulsed emission characteristics using a diode structure with a pulse-forming network under a vacuum of $\sim 5 \times 10^{-4} \mathrm{~Pa}$. The anode-cathode distance was $\sim 14 \mathrm{~cm}$. The half-value width of the pulsed voltage was $\sim 100$ ns. We measured the t-CNTs and p-CNTs under the same pulsed electric field to compare their emission characteristics.

\section{Results and discussion}

\subsection{Morphologies of t-CNTs}

Figure 1 shows the OM image of the pyramids. The distance between nearest-neighbor pyramids is $100 \mu \mathrm{m}$, and the length of bottom margin is $\sim 30 \mu \mathrm{m}$.

$\mathrm{KOH}$ is by far the most common anisotropic wet etchant. Its etch rates for single-crystal silicon depend on the crystallographic direction. For example, $\{111\}$ planes are etched about 100 times more slowly than are $\{100\}$ planes [13]. Therefore, the four oblique planes of a pyramid are in fact four $\{111\}$ planes, making a $54.7^{\circ}$ angle with respect to the horizontal (100) surface. For the $\sim 30-\mu \mathrm{m}$ bottom margin, we calculate the height of a pyramid to be $\sim 21.2 \mu \mathrm{m}$.

Because the mask used for lithography was made on a plastic film by a photoplotter, the shapes and precision of the designed square-dot array are not good, resulting in a pyramid array that is not very regular (Figure 1).

Figure 2 shows the SEM images of the t-CNTs. Figure 2(b) and (c) is the magnified images of the CNTs on a pyramid in (a). CNTs on the bottom flat are shown in Figure 2(d). We see from Figure 2 that the sample includes two kinds of morphologies: one is in the pyramid areas and the other the planar area. CNTs in the pyramid areas grew on the surface of the micro-pyramid array homogeneously, with left tussock-like CNTs with $2 \mu \mathrm{m}$ length and $\sim 20-50 \mathrm{~nm}$ diameters on the tips of the pyramids. The tussock-like CNTs are slanted with the pyramid surfaces, perpendicular to their substrates. The diameters of CNTs in the planar area are in the same range as those in pyramid areas. The range of CNT diameters in this study is in agreement with those in published reports $[12,14]$. Like those in references [12,14],

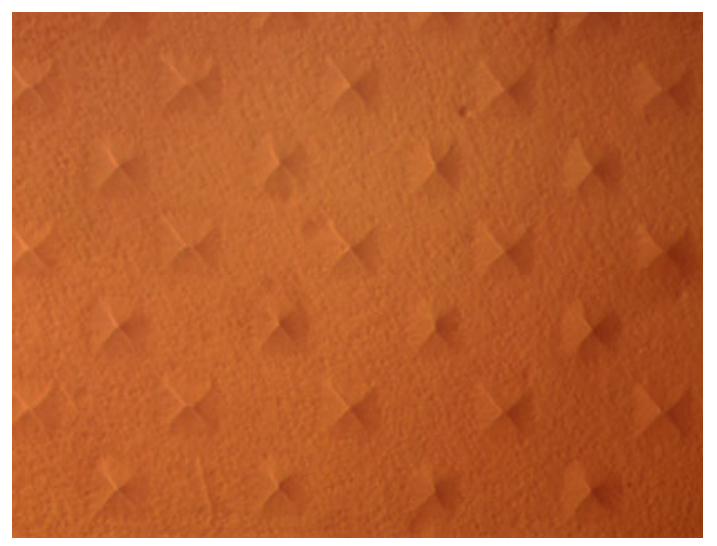

Figure 1 OM image of micro-pyramid array. The distance of nearest neighbor pyramids is $100 \mu \mathrm{m}$. 

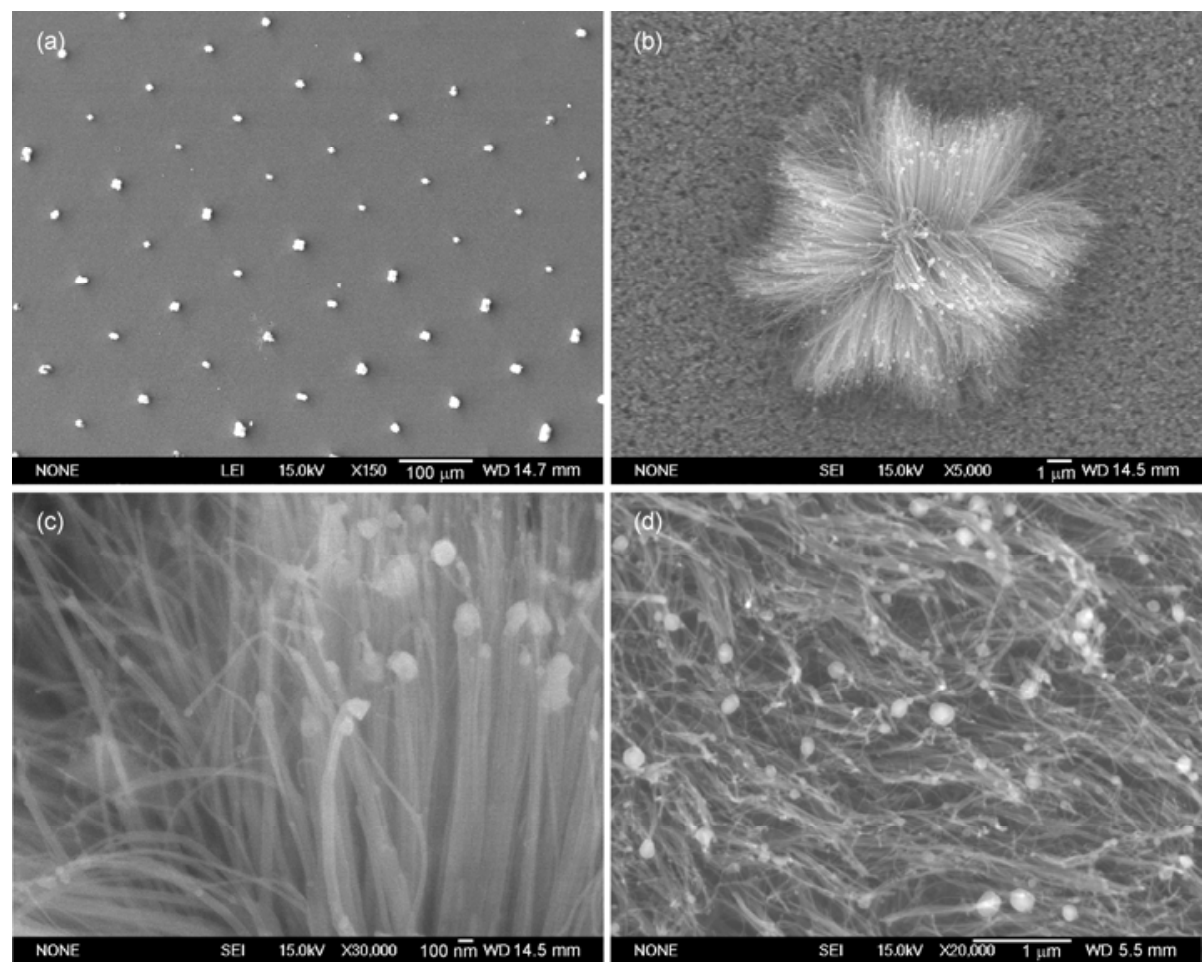

Figure 2 SEM images of CNTs grown on a tridimensional surface. (a) Total view; (b) CNTs grown on a pyramid tip; (c) magnified image of (b); (d) CNTs grown on the bottom flat.

the as-grown t-CNTs and p-CNTs are very dense, but there are some gaps in the t-CNTs due to the orientation-dependent CNT growth.

Our method of growing CNTs is the same as that in references [12,14], in which the results of different authors show good consistency. The reader who is interested in the details of CNTs prepared by the pyrolysis of FePc can see these references.

\subsection{Characteristics of intense pulsed emission in single- pulse mode}

Figure 3 shows the wave curves of the pulsed current and voltage vs. time, where (a) and (b) correspond to t-CNTs and p-CNTs and curves $\mathrm{C}_{1}$ and $\mathrm{C}_{2}$ correspond to the electric field and current, respectively. We see that the current curve agrees in general with the voltage. The peak of the displacement current, which is caused by the capacitor charge and discharge effects, cannot be found easily in Figure 3. Therefore, the capacitor charge and discharge effects can be neglected. The currents of $\mathrm{C}_{2}$ in Figure 3(a) and (b) are mainly from electron emission. For a peak field intensity of $\sim 12.2 \mathrm{~V} / \mu \mathrm{m}$, the peak emission currents and equivalent current densities are $324 \mathrm{~A}$ and $16.5 \mathrm{~A} / \mathrm{cm}^{2}$ for t-CNTs and $232 \mathrm{~A}$ and $12.1 \mathrm{~A} / \mathrm{cm}^{2}$ for $\mathrm{p}-\mathrm{CNTs}$. The emission current increases by $\sim 39.7 \%$ from the p-CNT to the t-CNT film. Points $\mathrm{A}$ and $\mathrm{B}$ are the emission turn-on and turn-off point; the turn-on and turn-off electric field can be deduced from $\mathrm{A}^{\prime}$ and $\mathrm{B}^{\prime}$. For the t-CNT film, the values of the turn-on and turn-off electric field and their mean value are 3.1, 2.7 and $2.8 \mathrm{~V} / \mu \mathrm{m}$, respectively. Those for the $\mathrm{p}-\mathrm{CNT}$ film are 5.0, 6.0 and $5.5 \mathrm{~V} / \mu \mathrm{m}$. Table 1 compares the intense pulsed emission characteristics of t-CNTs and p-CNTs.

\subsection{Mechanism of improving emission characteristics}

The improvement of emission characteristics for the micropyramid-array surface can be understood from two aspects. On one hand, the tridimensional surface increases the growth area of the CNT film, making more CNTs participate in electron emission. On the other hand, the micro-pyramid array suppresses the field-screening effect, resulting in a stronger electric field on the CNT emitters, which increases the emission current and decreases the turn-on electric field. Both aspects increase the emission of the t-CNT film, resulting in a stronger electron emission.

Although the growth area of the CNT film increases because of the tridimensional surface, the area of the tridimensional surface increases by only $\sim 15 \%$ over the planar surface in our case. Comparing with the $\sim 39.7 \%$ increase of the emission-current ratio, we see that more current increase comes from suppressing the field-screening effect.

However, the increase of $\sim 24.7 \%$ in emission current ratio, which is caused by suppressing the electric-field-screening effect, is not very distinct. This is because the quantity of pyramids per unit area is small. The basal area of the pyramids occupies only a small percentage, less than $10 \%$, of the total area of the planar substrate. In fact, most of the area of our 

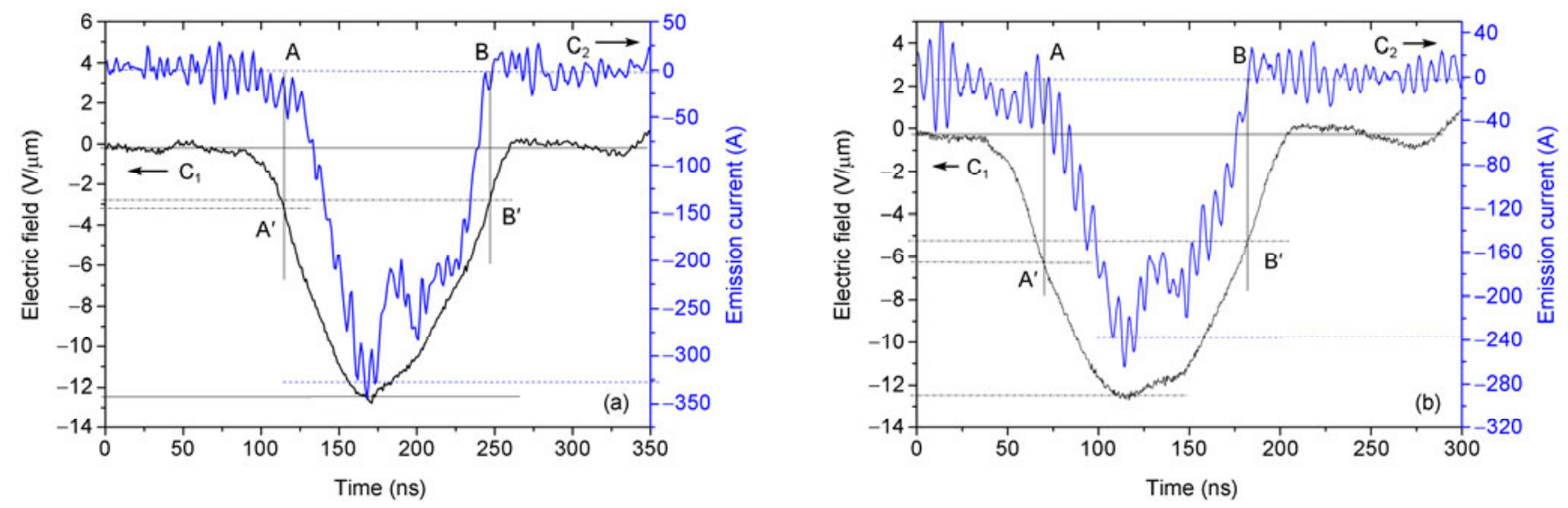

Figure 3 Waveforms of pulsed current and voltage corresponding to t-CNTs (a) and p-CNTs (b), in which $\mathrm{C}_{1}$ and $\mathrm{C}_{2}$ are electric field and current curves, respectively.

Table 1 Comparison between CNTs grown on tridimensional and planar surfaces

\begin{tabular}{cccc}
\hline Sample type & $\begin{array}{c}\text { Turn-on field } \\
(\mathrm{V} / \mu \mathrm{m})\end{array}$ & $\begin{array}{c}\text { Emission current } \\
(\mathrm{A})\end{array}$ & $\begin{array}{c}\text { Current density } \\
\left(\mathrm{A} / \mathrm{cm}^{2}\right)\end{array}$ \\
\hline $\mathrm{t}$-CNTs & 2.8 & 324 & 16.5 \\
$\mathrm{p}-\mathrm{CNTs}$ & 5.5 & 238 & 12.1 \\
\hline
\end{tabular}

t-CNT sample consists of planar-grown CNTs. The emission current of the t-CNT sample is the total of those from the pyramid and planar areas.

We think that the emission of a t-CNT film can be further improved by optimizing the size and density of pyramids on the substrate in designing the tridimensional surface. For example, we can increase the quantity of pyramids in a given area, control the size of pyramids according to their electric field distribution, and optimize them to a proper status to obtain a maximized emission current. Further research is going on, and the results will be reported later.

\section{Conclusion}

We presented a new scheme to suppress the field-screening effect by growing CNTs on a tridimensional surface. The intense pulsed emission characteristics of t-CNT and p-CNT films were measured using a diode structure in single-pulse mode. The even turn-on field decreased from $5.5 \mathrm{~V} / \mu \mathrm{m}$ for p-CNTs to $2.8 \mathrm{~V} / \mu \mathrm{m}$ for t-CNTs, and the peak emission current increased from $232 \mathrm{~A}$ for $\mathrm{p}$-CNTs to $324 \mathrm{~A}$ for t-CNTs at a peak field intensity of $\sim 12.2 \mathrm{~V} / \mu \mathrm{m}$. The peak current of $\mathrm{t}-\mathrm{CNT}$ increased by $\sim 39.7 \%$ over the p-CNTs.

This work was supported by the National Natural Science Foundation of China (51072184, 50972132, 51002143 and 60801022), the Aeronautical
Science Foundation of China (2009ZE55003 and 2010ZF55013) and the Basic and Advanced Technology Program of Henan (092300410139). The authors would like to thank Pan Haifeng from the Institute of Fluid Physics, Chinese Academy of Engineering Physics for help in measuring the intense pulsed emission characteristics.

1 Iijima S. Helical microtubules of graphitic carbon. Nature, 1991, 354: 56-58

2 De Heer W A, Chatelain A, Ugarte D. A carbon nanotube field emission electron source. Science, 1995, 270: 1179-1180

3 Bonard J M, Croci M, Klinke C, et al. Carbon nanotube films as electron field emitters. Carbon, 2002, 40: 1715-1728

4 de Jonge N, Bonard J M. Carbon nanotube electron sources and applications. Philos Trans R Soc Lond A, 2004, 362: 2239-2266

5 de Jonge N, Lamy Y, Schoots K, et al. High brightness electron beam from a multi-walled carbon nanotube. Nature, 2002, 420: 393-395

6 Teo K B K, Chhowalla M, Amaratunga G A J, et al. Fabrication and electrical characteristics of carbon nanotube-based microcathodes for use in a parallel electron-beam lithography system. J Vac Sci Technol B, 2003, 21: 693-697

7 Cheng Y, Zhang J, Lee Y Z, et al. Dynamic radiography using a carbon-nanotube-based field-emission X-ray source. Rev Sci Instrum, 2004, 75: 3264-3267

8 Choi W B, Chung D S, Kang J H, et al. Fully sealed, high-brightness carbon-nanotube field-emission display. Appl Phys Lett, 1999, 75: 3129_ 3131

9 Teo K B K, Minoux E, Hudanski L, et al. Microwave devices: carbon nanotubes as cold cathodes. Nature, 2005, 437: 968

10 Choi J H, Choi S H, Han J H, et al. Enhanced electron emission from carbon nanotubes through density control using in situ plasma treatment of catalyst metal. J Appl Phys, 2003, 94: 487-490

11 Kong B Y, Seon J Y, Lee S H, et al. Density control of highly populated carbon nanotubes grown by thermal chemical vapor deposition to improve their field emission characteristics. J Korean Phys Soc, 2004, 45: 1580-1583

12 Li D C, Dai L M, Huang S M, et al. Structure and growth of aligned carbon nanotube films by pyrolysis. Chem Phys Lett, 2000, 316: 349-355

13 Maluf N, Williams K. An Introduction to Microelectromechanical Systems Engineering. 2nd ed. London: Artech House, 2004

14 Song J H, Sun M Y, Chen Q, et al. Field emission from carbon nanotube arrays fabricated by pyrolysis of iron phthalocyanine. J Phys D: Appl Phys, 2004, 37: 5-9

Open Access This article is distributed under the terms of the Creative Commons Attribution License which permits any use, distribution, and reproduction in any medium, provided the original author(s) and source are credited. 